\title{
ISPCAN Child Abuse Screening Tools Retrospective version (ICAST-R): Delphi study and field testing in seven countries
}

\begin{abstract}
Objectives: To gain consensus among an ethnically and linguistically diverse group of international child protection experts on the structure and content of a new survey tool for retrospective measurement of child abuse, and to determine the performance of the instrument through an international field trial with young adults.

Methods: The questionnaire was developed through focus group discussions with international experts, and then subjected to a Delphi study in two waves to determine the perceived importance and translatability of items. The resultant questionnaire was translated into six languages and field tested in seven countries with convenient samples of young adults aged $18 і ̈ 26$ years $(\mathrm{N}=842)$.

Results: Child maltreatment experts from 28 countries provided input to questionnaire development. Satisfactory agreement on draft item inclusion and exclusion and the translatability of items was gained. The tool includes 15 primary questions about potentially abusive physical, sexual and emotional events, with follow-up questions about perpetrator characteristics, frequency of acts and periods in childhood when the recalled abuse occurred. The field test revealed lifetime prevalence per item usually exceeded 10\% (11/15 items; range $2.11149 .5 \%$ ). Internal consistency (Cronbach's alpha) was moderate to high for each of three item sub-sets (between .61 and .82) and the rates of missing data were low (less than $1.5 \%$ for 14 of 15 items). The great majority of respondents nominated either peer and/or adult perpetrators (between $82.3 \%$ and $98.2 \%$ depending upon the item), and among these, child/adolescent peers and non-family adults (including teachers for emotional and physical acts) were nominated often.
\end{abstract}

Conclusions: The ICAST-R is based on consensus from international experts, translates clearly and has satisfactory properties for adoption as a survey tool to estimate prevalence and describe perpetrators and other contextual aspects of child abuse.

Practice implications: This tool can be utilized in a broad range of cultures and languages and may contribute to improved research practice. Although the core items are limited to just 15 acts of maltreatment, if these behaviorally specific questions are adopted as key indicators and incorporated into comprehensive local, national or regional surveys, eventually there should be greater comparability in survey estimates.

Keyword: Child abuse; Retrospective questionnaire; Young adults; International field test 